\title{
A Rhodopsin Gene Expressed in Photoreceptor Cell R7 of the Drosophila Eye: Homologies with Other Signal-Transducing Molecules
}

\author{
Charles S. Zuker, Craig Montell, Kevin Jones, Todd Laverty, and Gerald M. Rubin \\ Department of Biochemistry, University of California, Berkeley, California 94720
}

\begin{abstract}
We have isolated an opsin gene from $D$. melanogaster that is expressed in the ultraviolet-sensitive photoreceptor cell R7 of the Drosophila compound eye. This opsin gene contains no introns and encodes a $\mathbf{3 8 3}$ amino acid polypeptide that is approximately $35 \%$ homologous to the blue absorbing ninaE and $R$ h2 opsins, which are expressed in photoreceptor cells R1-6 and R8, respectively. Amino acid homologies between these different opsins and other signal-transducing molecules suggest an important role for the conserved domains of rhodopsin in the transduction of extracellular signals.
\end{abstract}

Phototransduction, the neuronal excitation process triggered by light, provides an ideal model system for the study of sensory transduction in the nervous system. Rhodopsin, the major photoreceptor of both vertebrate and invertebrate eyes, consists of an apoprotein, opsin, covalently bound to a chromophore, generally 11-cis-retinal (reviewed in Fein and Szuts, 1982; Hargrave, 1982). Light activation of rhodopsin is the first step in the visual response, a biochemical cascade that converts the energy of an absorbed photon into a receptor potential (reviewed by Stryer, 1983, 1985; Kühn, 1984; Stieve, 1986). The chromophore is isomerized by light from the 11-cis to the all-trans configuration, which in turn leads to a conformational change in the opsin moiety. These photoactivated rhodopsin molecules then trigger the cascade of events that results in a transient change of the cation conductances of the photoreceptor cell membrane (reviewed by Fain and Lisman, 1981). Several vertebrate opsins have been sequenced (Ovchinnikov et al., 1982; Hargrave et al., 1983; Pappin and Findlay, 1984) and the genes for bovine and human opsins have been analyzed (Nathans and Hogness, 1983, 1984; Nathans et al., 1986). These vertebrate opsins are highly homologous in amino acid sequence and structure.

Drosophila is an attractive experimental organism in which to study signal transduction in the visual system using a combined molecular, genetic, and physiological approach (see, for

\footnotetext{
Received Sept. 26, 1986; accepted Nov. 25, 1986.

This work was supported by grants from the NIH to G.M.R. C.S.Z. is a fellow of The Jane Coffin Childs Memorial Fund for Medical Research. C.M. is supported by a NIH postdoctoral fellowship, and K.J. by an NSF predoctoral fellowship. We thank S. Mount, D. Kalderon, K. Moses, and J. Nathans for their comments. We also thank A. Tomlinson for his help with Figure 1, and K. Fryxell and E. Meyerowitz for communicating their results. We particularly want to thank Mark Stoneking and Alan Wilson for their help and advice in the construction of the phylogenetic trees.

Correspondence should be addressed to Charles S. Zuker, Department of Biology, B-022, University of California at San Diego, La Jolla, CA 92093.

Copyright (C) 1987 Society for Neuroscience $0270-6474 / 87 / 051550-08 \$ 02.00 / 0$
}

example, Pak, 1979; Hardie, 1983; Rubin, 1985). The compound eye of Drosophila contains 3 distinct classes of photoreceptor cells, $\mathrm{R} 1-6, \mathrm{R} 7$, and $\mathrm{R} 8$, distinguishable by their morphological arrangement and the spectral behavior of their corresponding visual pigments (reviewed by Hardie, 1983). In each of the approximately 800 ommatidia that make up the eye there are 6 outer (R1-R6) and 2 central (1 R7 and 1 R8) photoreceptor cells (Fig. 1). The photopigments found in the R1$\mathrm{R} 6$ cells, the $\mathrm{R} 7$ cell, and the $\mathrm{R} 8$ cell differ in their absorption spectra (Harris et al., 1976) most likely because different opsin genes are expressed in these distinct classes of photoreceptor cells. The 6 peripheral cells (R1-6) contain the major visual pigment, a rhodopsin that absorbs maximally at $480 \mathrm{~nm}$ (Ostroy et al., 1974). The gene encoding this visual pigment has been isolated by virtue of its homology to the bovine opsin gene and has been shown to correspond to the genetically defined ninaE locus (O'Tousa et al., 1985; Zuker et al., 1985). Of the 2 central photoreceptor cells, R7 contains a UV-sensitive pigment and R8 a blue nonadapting pigment (Harris et al., 1976; Hillman et al., 1983). We have previously reported the isolation and analysis of a Drosophila opsin genc that is transcribed specifically in the R8 photoreceptor cell (Cowman et al., 1986). We report here the isolation and analysis of a novel opsin gene that is homologous to the opsins expressed in the R1-6 and R8 pho-

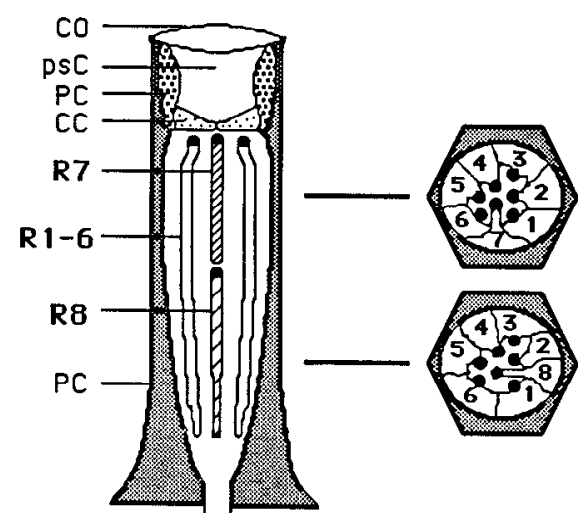

Figure 1. Adult ommatidial unit. The rhabdomeres (microvilli containing the visual pigments) of R1-R6 form an asymmetrical trapezoidal shape around the central rhabdomeres of the R7 and R8 cells. The R8 ccll is located below the R7 cell and extends through the proximal half of the retina. Inset shows cross sections through the distal (upper) and proximal (lower) regions of the retina. $C O$, corneal lens; ps $C$, pseudocone; $P C$, pigment cells; $C C$, cone cells; $R 1-6, R 7$, and $R 8$, photoreceptor cells. Adapted from Tomlinson (1985). 

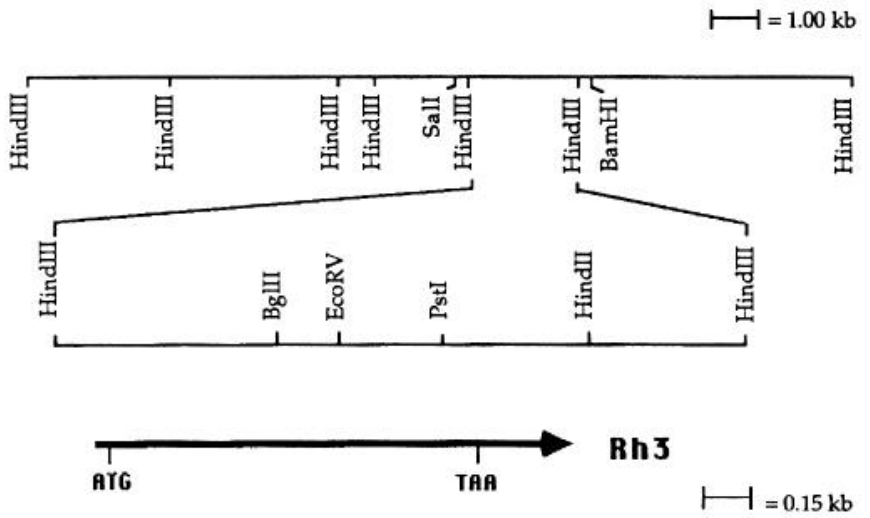

Figure 2. Restriction map of $\lambda \mathrm{DmRh} 3$ and structure of the RNA it encodes. Shown is a map of $\lambda \mathrm{DmRh} 3$ indicating the restriction sites for Hind III, Bam HI, and Sal I (top map). Also shown, in expanded view, is the region of the 2.4 kbase Hind III fragment encoding the RNA (lower map). The restriction sites for Eco RV, Pst I, Bgl II, and Hind II endonucleases are indicated. The diagram below the map shows the structure of the Rh3 RNA as deduced by comparison of the nucleotide sequences of a cDNA clone and the genomic clone, and S1 nuclease mapping and primer extension data (see Materials and Methods); note the lack of introns in Rh3.

toreceptor cells. We show that this opsin gene is transcribed in the UV-sensitive photoreceptor cell, R7, of the compound eye. In the accompanying paper (Montell et al., 1987), we describe the isolation and characterization of an additional opsin gene that is expressed in a nonoverlapping subset of R7 photoreceptor cells.

\section{Materials and Methods}

Isolation of $\operatorname{poly}(A)^{+} R N A$. RNA was extracted from the heads of the appropriate mutants, as described by O'Hare et al. (1983). Heads of adult flies (0-24 hr after eclosion) for Oregon R (P2), sev (sevenless; Harris et al., 1976), ora (outer rhabdomeres absent), and sev ora mutants (Harris et al., 1976) were separated from bodies as described by Oliver and Phillips (1970). Poly(A) ${ }^{+}$RNA was isolated by affinity chromatography on oligo(dT) cellulose columns (Blumberg and Lodish, 1980).

Blotting and hybridization of DNA and RNA. Fractionation of the RNAs on formaldehyde gels, transfer onto nitrocellulose paper, and prehybridization were carried out exactly as described by Chung et al. (1981). Hybridizations with nick-translated DNA probes were carried out at $65^{\circ} \mathrm{C}$ in $750 \mathrm{~mm} \mathrm{NaCl}, 100 \mathrm{~mm} \mathrm{NaH}_{2} \mathrm{PO}_{4}(\mathrm{pH} 6.8), 75 \mathrm{~mm}$ sodium citrate, $0.04 \%$ bovine serum albumin, $0.04 \%$ PVP- 40 , 0.04\% Ficoll, $0.5 \%$ SDS. Filters were washed in $0.2 \times \mathrm{SSC}(1 \times \mathrm{SSC}$ is $150 \mathrm{~mm} \mathrm{NaCl}$,
$15 \mathrm{~mm}$ sodium citrate), $0.5 \%$ SDS at $65^{\circ} \mathrm{C}$. The cDNA library (a gift from B. Yedvobnick and S. Artavanis-Tsakonas) was made from poly $(\mathrm{A})^{+}$ RNA isolated from the heads of adult flies ( $0-24 \mathrm{hr}$ after eclosion) of the Oregon R (P2) strain.

DNA probes. Nick-translation of DNA was carried out as described by Maniatis et al. (1982). All probes for in situ hybridization to head sections were made as described previously (Hafen et al., 1983).

Oligonucleotide probes. Two oligonucleotides were used as probes. The first [TATGTGCCIGAGGGTAA(C/T)CTGAC(C/T)] was 24 residues long, degenerate at positions 18 and 24 , contained inosine at position 9, and encoded the peptide YVPEGNLT. The second [CAGGCCAAGAAGATGAATGTCAA(A/G)TCCCTI] was a 30-residue oligonucleotide, degenerate at position 24 , contained inosine at position 30, and encoded the peptide QAKKMNVKSL. Synthetic oligonucleotides were purified by thin-layer chromatography on silica gel plates (Silica Gel $60 \mathrm{~F}$-254; EM Reagents) in $n$-propanol : $\mathrm{NH}_{4} \mathrm{OH}: \mathrm{H}_{2} \mathrm{O}$ (55:35:10), and were end-labeled with $\gamma^{-32}$ P-ATP, as described by Maniatis et al. (1982). Hybridizations with end-labeled oligonucleotide probes were carried out at $42^{\circ} \mathrm{C}$ in $7 \times \mathrm{SSC}, 0.1 \%$ bovine serum albumin, $0.1 \%$ PVP- $40,0.1 \%$ Ficoll. Filters were washed in $7 \times$ SSC, $0.5 \%$ SDS at $42^{\circ} \mathrm{C}$. Four genome equivalents of a total genomic library (Maniatis et al., 1978) were screened.

In situ hybridization to tissue sections. Preparation of $8 \mu \mathrm{M}$ frozen sections of adult heads and hybridization of ${ }^{3} \mathrm{H}$-labeled probes were as described by Hafen et al. (1983), except that the acid and pronase treatments were omitted in the pretreatment of the slides.

DNA seyuence analysis. DNA sequencing was carried out on randomly sheared fragments according to the chain termination procedure of Sanger et al. (1977). M13 mp18 and mp19 were used as sequencing vectors and TG1 (the gift of Toby Gibson, MRC Laboratory of Molecular Biology, Cambridge, England) as the host strain. Reactions were carried out as described by Bankier and Barrell (1983) with $\alpha{ }^{-35}$ S-dATP as the radioactive nucleotide. The genomic sequence of the $R h 3$ opsin gene was determined over both strands. The complementary DNA (cDNA) sequence was determined on 1 strand.

Primer extension and S1 nuclease analysis. Primer extensions were carried out by hybridizing $5 \mathrm{ng}$ of a synthetic 20 base oligonucleotide (complementary to positions +18 to +37 ) in separate $20 \mu$ l reactions to either an M13 clone containing the 0.75 kbase Hind III- $B g l$ II fragment of $\lambda$ DmRh3 (see Fig. 2), $20 \mu \mathrm{g}$ of head poly(A)+ RNA, or $20 \mu \mathrm{g}$ of body poly $(\mathrm{A})^{+}$RNA. Reverse transcription was then carried out as described for cDNA synthesis by Maniatis et al. (1982). S1 nuclease protection experiments were carried out as described by Maniatis et al. (1982). Single-stranded DNA probes used in the S1 endonuclease protection experiments were prepared by synthesizing a radiolabeled second strand on M1 3 templates. The newly synthesized material was separated from the template after restriction enzyme cleavage by boiling in $30 \%$ dimethyl sulfoxide (DMSO) and gel purified on a $1 \%$ agarose gel.

In situ hybridization to polytene chromosomes. Polytene chromosome squashes (Canton S strain) were prepared as previously described (Zuker et al., 1985). Hybridization with biotinylated DNA probes was carried out according to Langer-Sofer et al. (1982) with the following modifications: DNA was nick-translated using Bio-16-dUTP (Enzo Biochem)

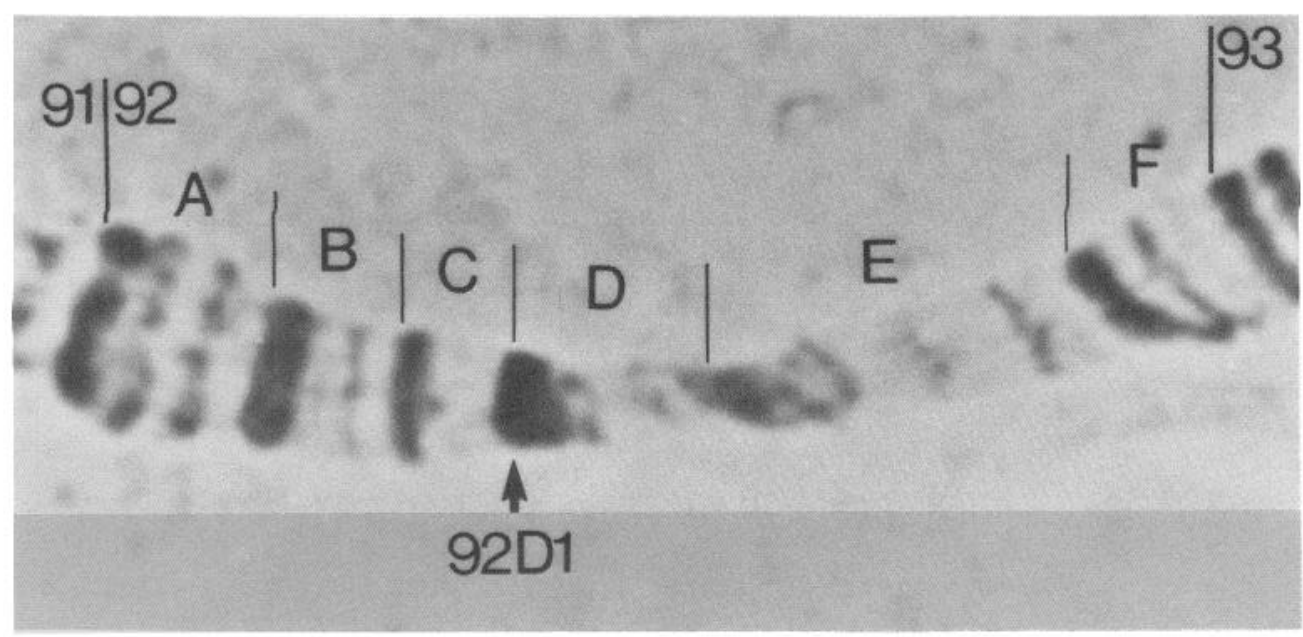

Figure 3. In situ hybridization to salivary gland chromosome squashes. $\lambda \mathrm{DmRh} 3$ was biotinylated as described in Materials and Methods and used as a hybridization probe to determine its chromosomal location. Shown is the 92 region of chromosome III of Drosophila melanogaster (Canton S). The arrow indicates the site of hybridization at 92D1; no other sites of hybridization were observed. 


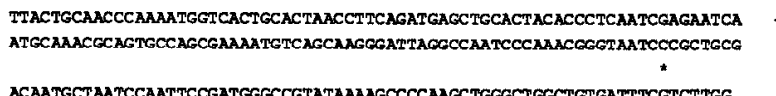

ACAATGCTAATCCAATTCCGATGGGCCGTATAAAAGCCCCAAGCTGGGCTGGCTGIGATTTCGTCTTEG

CCCGCAGACCGGAGC ATG GAG TCC GGT AAC GTG TCG TCG AGT CTG TTT GEC AAC GTG MET Glu Ser Gly Asn Val Ser ser sar Leu phe Gly Asn Val

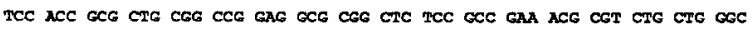
ser Thr Ala Ieu Arg Pro Glu Ala Arg Leu Ser Ala Glu Thr Arg hau Lou Gly

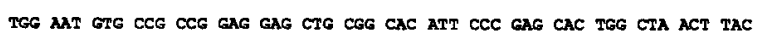
Tro Asn val pro Pzo Glu Glu Lau Arg Hia Ile Pro Glu Els Trp Lau Thr Tyr

CES GAG CCG CCC GAA TCG ATG AAC TAC CTG CTG GGC ACG CTC TAC ATC TTC TTC

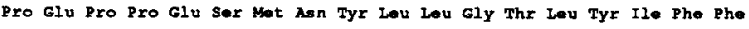

ACC CIG ATG TCG ATG CTG GGC AAT GGA CTG GTG ATT TGG GTC TTC TCC GCA GCC Thr Leu Met Sar Mat Leu Gly asn Gly Lau Val Ile Tro Val phe Sar Mla Ala

AAA TCG CTG CGA ACT CCC TCC AAT ATA CTE GTC ATC AAT CTG GCC TTC TGC GAC Lys Ser Leu Arg Thx Pro Ser Asn Ile leu Val Ite Asn Leu Ala Phe Cys Asp

TTC ATG ATG ATG GTC Aag ACt CCG ATA TTC ATC TAC Aat AGC TTC CAC CAG GGa phe met wet met Val Lys Thr Pro Ile phe Ile Tyr Asn Ser Phe His Gin Gly

TAT QCE CTG GGT CAT CTG GGA TGC CAG ATC TTT GGA ATC ATT GGC TCE TAT ACG Tyr Ala leu Gly Bly Leu Gly Cys Gln Ile Phe Gly Ile Ile Gly Ser Tyr Thx

GGA ATC GCT GeC GET GeC ACC AAT GEC TTT ATA GCe TAC GAT OCA TTC AMT GTC Gly Ile Ala Ale gly ala thr asn Ala Phe Ile Ala Tyr Asp Arg phe Asn val

ATC ACT CGA CCC ATG GAG GGC AAG ATG ACG CAT GGC AAG GCC ATT GCC ATG ATC Il. Thr Arg Pro met Glu Gly lys mat Thr gis Gly bys Ala Ile wal thet Ile

ATA TTC ATC TAC NTG TAC GCC NCT CCA TGG GTG GTT ECC TGC TAC ACG GAG ACT

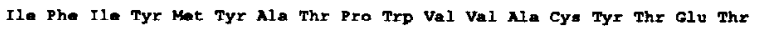
TGG GGC CGT TTT GTG CCG GAG GGA TAT CTG ACA TCC TGC ACC TTT GAC TAT CTC Irp Gly arg phe val pro Glu Gly Thr Lau Thr ser Cya Thr Phe Asp Tyr Lau

ACC GAT AAC TTC GAT ACG CGA CTC TTT GTG GCC TGC ATC TTC TTC TTC AGC TTC Thr Asp Asn Phe Asp Thr Arg lou phe val Ala Cys Ile the Phe Phe ser Phe

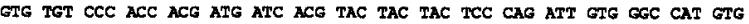
val cys pro Thr thr mat Ile Thr Tyr tyr Tyr ser Gln Ile val Gly Bls VaI

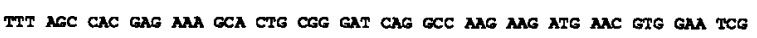

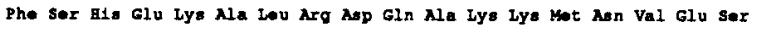

CTG CGT TCG AAT GTG GAC AAA AAC AAG GAG ACG GCG GAX ATC CGG ATA GCC ANX Lou Arg Ser Asn Val Asp Lys Asn Lys Glu Thr Ala Glu Ile Arg Ile Ala Lyy

GCG SCC ATC ACC ATA TGC TTC CTG TTC TTP TGC TCG TGE ACG CCG TAC GGA GTH ala dla Ile Thr Ile Cys phe leu Phe Phe cya ser Trp Thr Pro Tyr gly Val

ATG TCG CTG ATT GGC GCC TTT GGG GAT AAG ACC CTT TTG ACG CCC GGA GCC ACA Mat Sar Leu Ilo gly Ala phe Gly Aap hys Thr leu lau Thr pro Gly kla Thr 32

ATG ATT CCC GCC TGT GCC TGC AMA ATG GTG GCC TGC ATC GAT CCG TTC GTG TAC 1036 Mat Ile pro Ala Cys Ala Cys lya mat Val Mla Cya Ile Asp pro phe val Tyr 338

GCC ATA AGT CAT CCC AGA TAC CGC ATG GAG CTG CAg Na CGA TGT CCC TGG CTG 1090 Ala Ile Ser H1s Pro Arg Tyr Arg Mat Glu Iou Gln Lys Arg Cys Pro Trp Leu 356

GCG CTC AAC GAA AAG GCG CCG GAA TCG TCG GCT GTC GCC TCC ACC AGC ACC ACC 1144 Ala Lou Asn Glu Lys Ala Pro Glu Ser ser Ala val Ala ser Thr Ser Thr Thr 374

CAG GAA CCA CAG CAG ACE ACC GCC GCC TAM TGCANATTCCCACCCAACAGCCACCCATCC 1204 Gin Glu gro Gln Gln Thr Thr Ala Ala

AAAAAATGCTGACAATGATGATGAACTGAAACGGCAACGAACTCGTAATACCAAACGCCAGGGAAMATTE $\quad 1274$ TATAAATATT TCACGGAGTTTCTGCTAGAGTGCAAAGAGTTGAAAATATTTTCTTGTTATGATTTGTAGT 1344 CACTTTTGTGTTTATGAAAGAAATATAGTAAAATTACAGCAAGCTATGGAAAAAAGCGTTATATAAACTG 1414

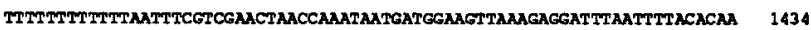

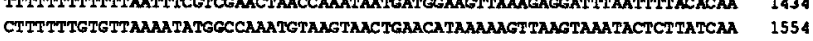
CTGAATAAGTCATTAACTCAGT TEACAACTITAATAAAATAABTTCTPAAAAATCTIGAGTPGATAMAAT 1624 CAGGTCATACTCAACGCATTGTGCAAAACACCTATTGTTTCACCAGGTTTACTTATGITTATAGAAATCC 1694

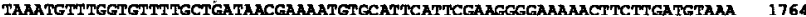
M TGCAGGGCACTCAGACGAGCCGAMGAAGGCGGCTTAATGCTCCCAGGAGCGGAGATGGCCAMCAGCAAAA 1904 $\begin{array}{ll}\text { TGCAGGGCACTCAGACGAGCCGAAGAAGGCGGCTTAATGCTCCCAGGAGCGGAGATGGCCAACAGCAAAX } & 1904 \\ \text { GAGTTGGCCAAGAACAATAGGGAATTGTGCCAGTPCCAATGGAATCCACAGACGGGAAAGGAGCTCTCTA } & 1974\end{array}$ ACTCTCTTGTGCTCGCTTACTTTCTCTGGAGAGCGAAAGCTT

Figure 4. Nucleotide sequence and deduced amino acid sequence of Drosophila Rh3 opsin. The sequence shown was determined on both strands of the genomic clone (2.4 kbase Hind III fragment; see Fig. 1). The region from nucleotide 400 to 1270 was also sequenced in a cDNA clone. The underlined region at -28 to -34 shows the putative TATA box. The star at nucleotide +1 indicates the position of the start of transcription, as determined by primer extensions and $S 1$ nuclease mapping data. The putative poly $(\AA)$ addition signal is underlined at position and hybrids were detected using a Detek-I-HRP detection kit (Enzo Biochem).

\section{Results and Discussion}

\author{
Isolation of $\lambda D m R h 3$, a DNA segment encoding a novel \\ Drosophila opsin
}

The different spectral sensitivities of the various photoreceptors of the Drosophila eye indicate the presence of multiple rhodopsins (reviewed in Hardie, 1983). The gene encoding the opsin expressed in the 6 peripheral photoreceptor cells (R1-6) has been isolated by virtue of its sequence homology to the bovine rhodopsin gene (O'Tousa et al., 1985; Zuker et al., 1985). This gene corresponds to the genetically identified ninaE locus (Scavarda et al., 1983). We have used the ninaE gene to search for cross-homologous sequences in the Drosophila genome and thereby isolated the gene encoding the opsin expressed in the central, blue-absorbing R8 photoreceptor cells ( $\mathrm{Rh} 2$ opsin; Cowman et al., 1986); neither the ninaE nor the Rh2 opsin genes appear to be expressed in the central R7 photoreceptor cell (Zuker et al., 1985; Cowman et al., 1986). Therefore, at least one additional rhodopsin gene, the one encoding the opsin expressed in the R7 photoreceptor cell, must exist in the Drosophila genome. Low-stringency hybridizations of Drosophila genomic and cDNA libraries with the cloned ninaE and $\mathrm{Rh} 2$ probes did not reveal any additional homologous sequences. Assuming that functionally significant regions of rhodopsin might be conserved among the different opsin genes, we designed oligonucleotide probes (see Materials and Methods) corresponding to 2 of the most highly conserved regions between ninaE and $\mathrm{Rh} 2$ opsins in order to screen a genomic library with greater sensitivity. One of these regions encodes an 8 amino acid sequence that is conserved between the 2 Drosophila and bovine rhodopsins (Nathans and Hogness, 1984; O'Tousa et al., 1985; Zuker et al, 1985; Cowman et al., 1986). The other encodes a 10 amino acid sequence that is unique to Drosophila opsins (Zuker et al., 1985; Cowman et al., 1986); vertebrate opsins làck this 10 amino acid region. Positive clones were isolated and counterscreened with radiolabeled ninaE and $\mathrm{Rh} 2$ gene-specific probes to eliminate the ninaE and $\mathrm{Rh} 2$ cognate sequences. Three of the genomic clones that hybridized strongly with both oligonucleotide probes represented overlapping sequences, hereafter referred to as Rh3. Homology to the oligonucleotides was confined to a 2.4 kbase Hind III fragment (Fig. 2).

The ninaE and $\mathrm{Rh} 2$ genes have been cytogenetically mapped to chromosomal positions $92 \mathrm{~B} 8-11$ and $91 \mathrm{D} 1-2$, respectively (Zuker et al., 1985; Cowman et al., 1986). The Rh3 clones were mapped by in situ hybridization to salivary gland chromosomes to chromosomal position 92D (Fig. 3). This cytogenetic location corresponds to that of $\lambda 512$, a genomic clone isolated by Levy et al. (1982) on the basis of its encoding a head-specific transcript. No mutations affecting vision or visual input-mediated behavior have been isolated at or near this cytogenetic location.

\section{$R h 3$ encodes an opsin}

We used the 2.4 kbase Hind III fragment of $\lambda \mathrm{DmRh} 3$ (see Fig. 2) to screen a Drosophila cDNA library and isolated several

1589-1599 (note the presence of 2 overlapping AATAAA consensus sites). The Rh3 opsin protein sequence is shown aligned under the nucleotide sequence. 


\section{Cytoplasmic}

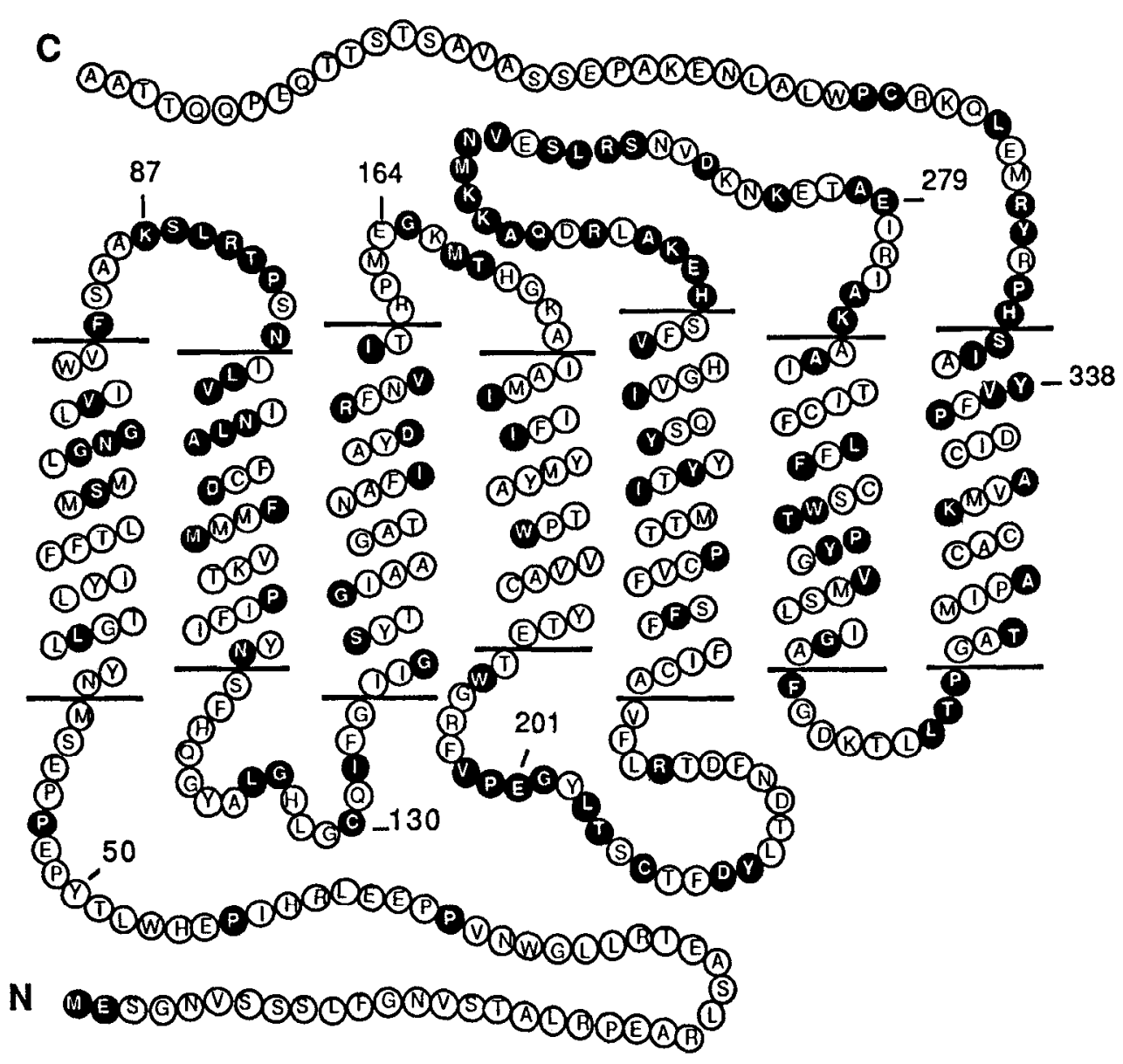

Extracellular
Figure 5. Proposed structure of Drosophila $\mathrm{Rh} 3$ opsin. Drawing is a modified version of the model of Hargrave (1982) and Ovchinnikov (1982). Putative transmembrane domains were determined by the algorithm of Kyte and Doolittle (1982). Amino acid residues are indicated by their single letter codes. Black solid circles indicate identities among the Drosophila ninaE, Rh2, and Rh3 opsins.
cDNA clones. Using M13 dideoxynucleoside triphosphate sequencing, we have determined the DNA sequence of one of those cDNA clones and of the 2.4 kbase Hind III genomic fragment of $\lambda \mathrm{DmRh} 3$ (see Fig. 2). The genomic sequence we characterized included the same coding region as that present in $\lambda 512$ (C. Montell and G. M. Rubin, unpublished observations; K. Fryxell and E. Meyerowitz, personal communication). Figure 4 shows the nucleotide sequence and the deduced amino acid sequence of the Rh3 gene. The structure of the RNA (Fig. 2) and the position of the start of transcription were determined by primer extension and S1 nuclease mapping analyses (data not shown).

Rh3 encodes a 383 amino acid polypeptide that shares 130 and 125 amino acid identities with the ninaE and $\mathrm{Rh} 2$ opsins, respectively; 116 of these residues are conserved between all 3 polypeptides. In addition, the protein encoded by $\mathrm{Rh} 3$ contains all of the structural features expected of a visual pigment protein: 7 hydrophobic domains separated by hydrophilic sequences, a presumed retinal-binding site in the seventh transmembrane domain (Lys 328), a series of potential phosphorylation sites (Ser and Thr residues) in the C-terminal region of the polypeptide chain, and a glycosylation site(s) in the extracytoplasmic face (Asn 5 and Asn 13). This is the only Drosophila opsin that contains more than 1 potential $\mathrm{N}$-linked glycosylation site (consensus, Asn-X-Ser, Asn-X-Thr). Figure 5 shows the proposed structure of the Rh3 opsin, based on the algorithm of Kyte and Doolittle (1982) and the models of Ovchinnikov (1982) and Hargrave (1982). It is worth noting that amino acid residues 198-205 and 258-267, corresponding to oligonucleotide probes 1 and 2 , are $71 \%(5 / 7)$ and $90 \%(9 / 10)$ conserved in Rh3 (see Materials and Methods). At the nucleotide level, oligonucleotide 1 shows $83 \%$ identity (20/24, including degenerate residues) with the corresponding region of the $\mathrm{Rh} 3$ gene, and the oligonucleotide 2 sequence is $86 \%$ conserved (26/30, including degenerate residues).

\section{$R h 3$ is expressed in the UV-sensitive R7 photoreceptor cells}

Previous comparision of the amino acid sequences of the ninaE opsin with the Rh2 opsin revealed a high degree of homology (67\%; Cowman et al., 1986). Both of these opsins have absorption maxima in the blue region of the spectra (reviewed by Hardie, 1983). In contrast, Rh3 is only approximately $35 \%$ homologous with the ninaE and Rh2 proteins (Fig. 5). In order to determine in which photoreceptor cell type the Rh 3 opsin is expressed, and thus what spectral behavior it mediates, we examined the levels of mRNAs homologous to this gene in mutant 
Figure 6. Expression of $\mathrm{Rh} 3$ transcripts. Poly $(\mathrm{A})^{+}$RNAs were extracted at different stages of development, from adult heads of wild-type, sev, and sev ora flies, and from wild-type adult bodies. The RNAs $(2.0 \mu \mathrm{g} /$ lane $)$ were gel fractionated, blotted, and hybridized to a DNA probe derived from nucleotides 1070-2016 of Rh3, as described in Materials and Methods. Lane E, embryos; lanes $1,2,4,6$, and 8 , days of development; $A$, newly eclosed adults. $\lambda$-Hind III and $\Phi$ X174 Hae III fragments were used as size markers.

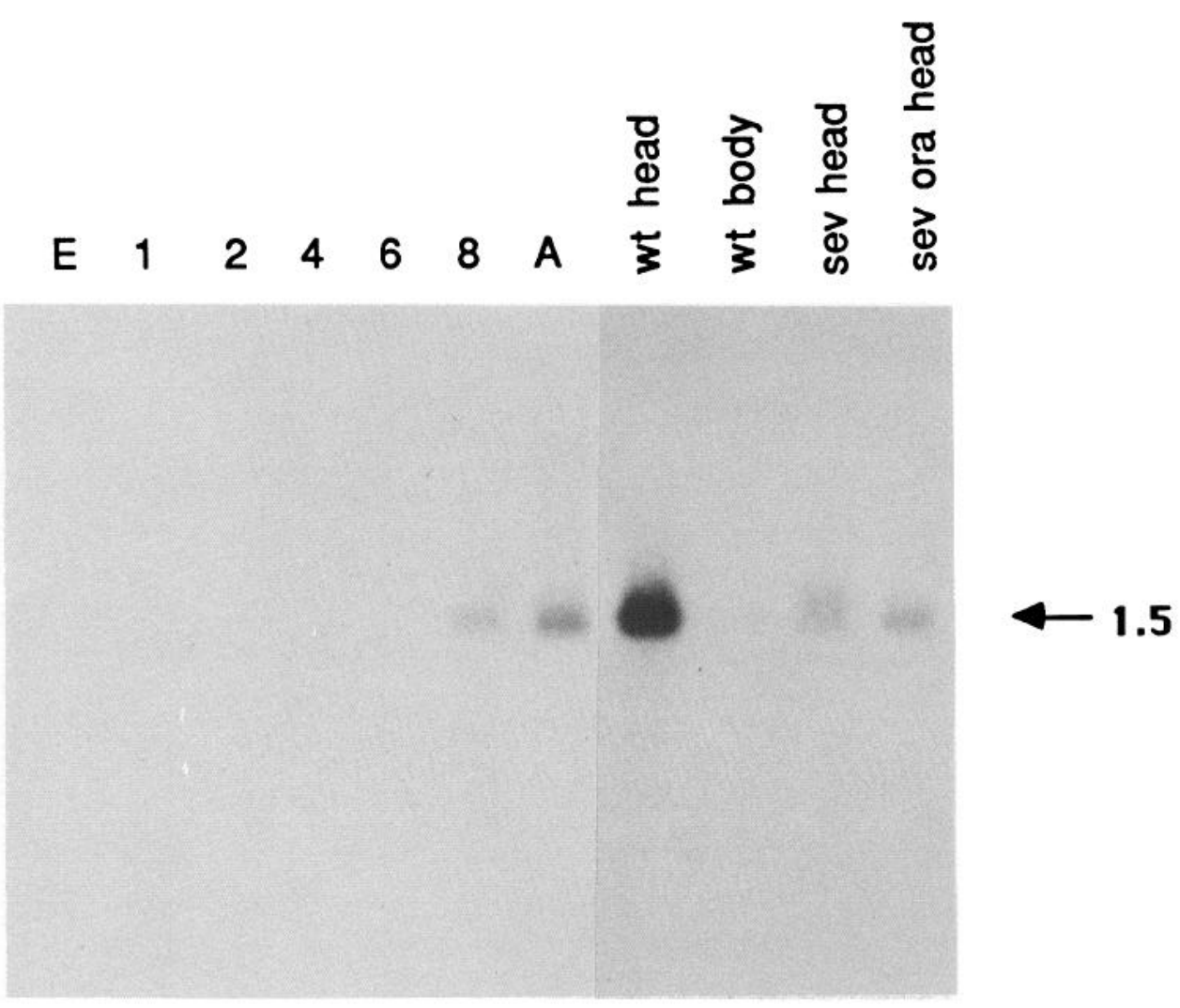

(outer rhabdomeres absent) flies, which lack the 6 peripheral outer photoreceptor cells (R1-R6), and from the double mutant sev ora, which has only the central R8 photoreceptor cells (lacking R1-6 and R7). The RNAs were fractionated on agaroseformaldehyde gels and hybridized to a radiolabeled DNA fragment consisting of the $3^{\prime}$ region of Rh3 (nucleotides 1070-2016; Fig. 4). This sequence does not hybridize to any of the other Drosophila opsin genes. Rh3 hybridizes to a 1.5 kbase RNA
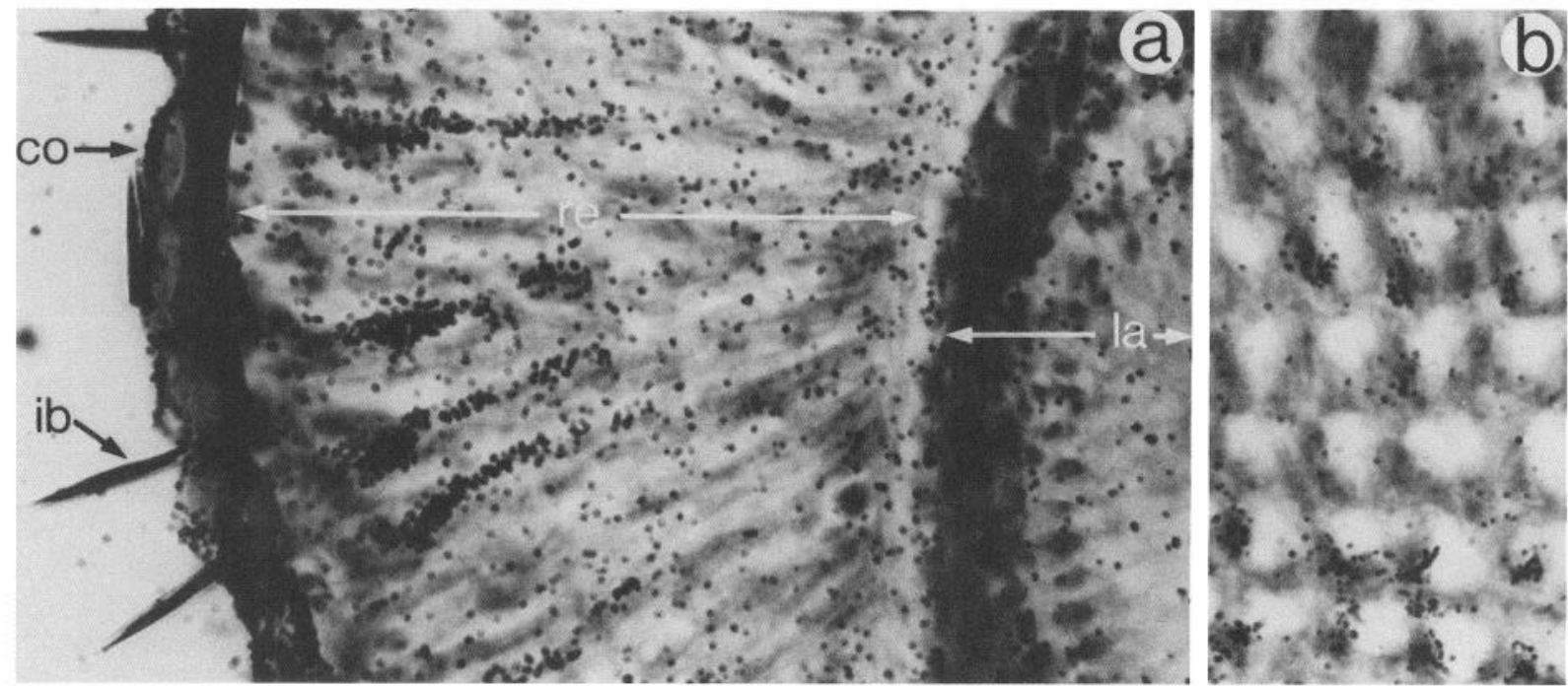

Figure 7. Spatial distribution of Rh3 transcripts in tissue sections of adult heads. Shown is the hybridization of an Rh3-specific probe to adult retinas (exposure, $30 \mathrm{~d}$ ). Longitudinal $(a)$ and tangential $(b)$ sections of adult heads are shown. The R7 cell is located above the R8 cell and extends through the distal half of the retina (see Fig. 1). Re, retina; $l a$, lamina ganglionaris; $c o$, corneal lens; $i b$, interommatidial bristles. 


hGreen
hred
bBlne
hrhodope1n
ha B-AR
Dm Rh1
Dm Rh2
Dn Rh3
D. Rh4

MAQQWS LQRLAGRHPQDSYEDSTQSSIFTYTNSNSTRGPFEGPNYH IAPRWVMAQQWS LQRLAGRHPQDS YED STQSSIFTYTNSNSTRGPFEGPNYH IAPRWVMRKMSEEEFYLFKNISSVGPWDGPOYHIAPVWAMNGTEGPNE YVPF SNATGWVRSPFEYPQYYLAEPWQMGPPGNDSDFLLTTNGSHVPDHDVTEERDEAWVV ME SFAVAAAQLGPHFAPLS-NGSVVDKVTPDMAHLI SPYWNOFPAMDP IW-MERSHLPETPFDLAHSGPRFQAQSSGNGSVLDNVLPDMAHLNVPYWSRFAPMDPMM-ME SGNVS SSLF GNVSTALRPEARLSAETRLLG--WNVPPEELRH TP-EHWLTYPEPPESM MEPLCNASEPP LRPEARSSGNGDLQF LGWNVP PDQIQY IP-EHWLTQLEPPASM

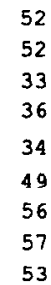

52

36

34

49

\begin{tabular}{l|l|l|l|l|l} 
Rh3 & NYLLGTIYIFFTLMSMLGNGLVIWV & ESAAKSLR--TPSN & ILVINLAF CDFMMM-VKTP IF IYN & SF FQGYALGHLGCQIFG \\
Rh4 & HYMLGVFYIFLFCASTVGNGMVIWI & FSTSKSLR--TPSN & MFVLNLAVFDLIMC-LKAPIFIYN & SFHRGFALGNTWCQIFA
\end{tabular}

7
9
56
5
5

1
4
10
4
4
4

8
0
9
0
5
2
8

\begin{tabular}{|c|c|c|c|c|c|}
\hline$-\infty-\infty$ & MVVMV-LAFCF CWGPYAFFACFAA & ANP GYPFHP-- & LMAALPAFFAKSATIYNPVIYVFM & NRQFRNCILQ---L & 336 \\
\hline & MVVVMI-FAYCVCWGPYTFFACFAA & ANP GYAF HP-- & LMAALPAYFAKSATIYNPVIYVFM & NRQFRNCILQ- - L & 336 \\
\hline$\cdots$ & MVVVMV-GSFCVCYVPYAAF AMYMV & NNRNHGLDL-- & RLVTIPSFF SKSACIYNP I IYCEM & NKQFQACAMK--MV & 318 \\
\hline$-m--$ & MVI IMV-IAFLICWVPYASVAFYIF & THQGSNFGP-- & IFMTIPAFFAKSAAIYNPVIYIMM & NKQF RNCMLT-TIC & 322 \\
\hline KEHKAL & KTLGI IMGTFTLCWLPFF IVNIVHV & --IQDNLIPKE & VYILLNWLGYVNSA-FNPLIYCRS & -PDFRIAFOELLCL & 342 \\
\hline---- & VALVTITLWFMA-WTPYLVINCMGL & F-KFEGLTP-- & LNT IWGACFAKSAACYNPIVYGIS & HPKYRLALKE-KCP & 345 \\
\hline & VALTTISLWFMA-WTPYLVICYFGL & F-KIDGLTP-- & LTTIWGATEAKTSAVYNPIVYGIS & HPKYRIVLKE-KCP & 352 \\
\hline----- & AAITICFLFECS-WTPYGVMSLIGA & FGDKTLLTP-- & GATMIDACACKMVACIDPFVYAIS & HPRYRMELQK-RCP & 354 \\
\hline--- & AAITICFLFFVS-WTPYGVMSLIGA & FGDKSLLTP-- & GATMIPACTCKLVACIDPFVYAIS & HPRYRLELQK-RCP & 350 \\
\hline & $\star \star$ & & & & \\
\hline \multicolumn{5}{|c|}{ FGKKVDDGSEI-SSASKTEVSSVSSVSPA } & 364 \\
\hline \multicolumn{5}{|c|}{ FGKKVDDGSEL-SSASKTEVSSVSSVSPA } & 364 \\
\hline \multicolumn{5}{|c|}{ CGKAMTDESDTCSS-QKTEVSTVSSTQVGPN } & 348 \\
\hline \multicolumn{5}{|c|}{ CGKNPLGDDEASATVSKTETSQVAPA } & 348 \\
\hline \multicolumn{5}{|c|}{ RRS S SKAYGNGY S SNGNGKTDYMGEASGCQLGQEKESERLCEDPPGTE SFVNCQGTVP SLS LD SOGRNCS TNDSPL } & 418 \\
\hline \multicolumn{5}{|c|}{ CCVFGKVDD-GKS SDAQSQ-ATASEAESKA } & 373 \\
\hline \multicolumn{5}{|c|}{ MCVFGNTDE-PKPDAPASDTETTSEADSKA } & 381 \\
\hline \multicolumn{5}{|c|}{-WLALNEKA-PESSAVAS-TSTTQEPQQTTAA } & 383 \\
\hline \multicolumn{5}{|c|}{-WLGVNEKSGEIS SAQS---TTTQEQQQTTAA } & 378 \\
\hline
\end{tabular}

Figure 8. Amino acid sequence homologies between the $\beta$-adrenergic receptor and the human and Drosophila opsins. Colinear alignment of the deduced amino acid sequences of the 4 human visual pigments (Nathans and Hogness, 1984; Nathans et al., 1986), the hamster $\beta$-adrenergic receptor (Dixon et al., 1986), and the 4 Drosophila opsins (O'Tousa et al., 1985; Zuker et al., 1985; Cowman et al., 1986; Montell et al., 1987). Amino acids are designated by their single letter codes. Alignment has been optimized for the largest number of identities with the least number of gaps. Boxed areas show the position of the 7 putative transmembrane domains of Drosophila opsins. Stars above the sequence indicate homologies between the $\beta$-adrenergic receptor and a minimum of 4 opsin genes. The arrowhead indicates the position of the lysine residue to which the retinal chromophore is thought to be bound. 
Figure 9. Phylogeny of visual pigments and the $\beta$-adrenergic receptor. This phylogenetic tree relating the different animal visual pigments and the $\beta$-adrenergic receptor was constructed on the basis of the principlc of minimal mutation distances (parsimony principle). The data presented in Figure 8 were subjected to phylogenetic analysis by the Farris (1972) method. The most parsimonious tree is shown. Branch lengths (number of mutational events) are indicated in the scale below the tree. $D m-R h I$ stands for the Drosophila ninaE gene, Dm-Rh2, Dm-Rh3, and $D m-R h 4$ refer to the Drosophila $\mathrm{Rh} 2$, $\mathrm{Rh} 3$, and Rh4 opsin genes. The hamster $\beta$-adrenergic receptor (Dixon et al., 1986) is referred to as $h a-\beta-A R$. $h$-Green, $h$-Red, and $h$-Blue refer to the human color opsins, and $h$-Rhod to human rhodopsin (Nathans and Hogness, 1984; Nathans et al., 1986).

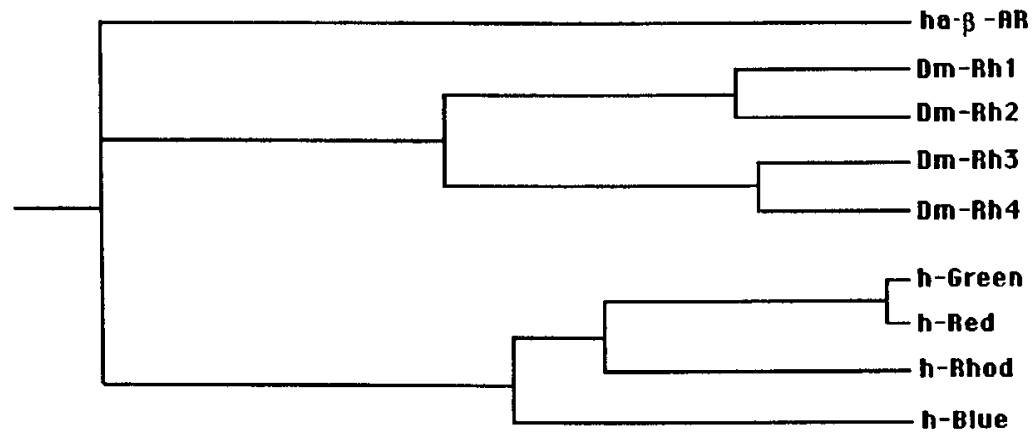

species that accumulates in the late pupa and peaks after eclosion (Fig. 6). This RNA is present in the heads of wild-type flies or in flies carrying the ora mutation (data not shown). However, the RNA is greatly reduced in flies carrying the sevenless mutation (either sev or sev ora). The small amount of hybridization observed in the heads of sev and sev ora flies may represent expression of this gene in other cell types, as this RNA is also present in the heads of mutant flies lacking all photoreceptor cells of the eye (glass; data not shown). The spatial distribution of Rh3 transcripts was directly determined by hybridizing the radiolabeled $\mathrm{Rh} 3$ gene-specific probe to tissue sections of wildtype adult heads. Figure 7 shows that RNAs homologous to Rh3 are localized to the distal region of the retina; this is the location of the UV-sensitive R7 photorcceptor cclls (scc Fig. 1). This result, taken together with the severe reduction of $\mathrm{Rh} 3$ transcripts in mutants carrying the sev mutation (Fig. 6), indicates that the Rh 3 opsin is transcribed in the central R7 photoreceptor cells of the compound eye.

The spectral and dichroic properties of the Drosophila R7 photoreceptor cells have not been studied in detail. These cells contain a bistable pigment system with sensitivity in the UV (330-350 nm) and metarhodopsin forms in the blue region of the spectrum (see Hardie, 1983; Hillman et al, 1983). We believe the differences between the primary sequences of the ninaE and $\mathrm{Rh} 2$ opsins, and the much more divergent $\mathrm{Rh} 3$ opsin, reflect the different spectral properties of these photopigments (see Montell et al., 1987).

\section{Drosophila opsins contain amino acid sequence domains that are highly conserved with other signal-transducing proteins}

Vertebrate and invertebrate rhodopsins interact with at least 2 cytoplasmic proteins: a G-protein (transducin) and rhodopsin kinase (reviewed by Kühn, 1984; Stieve, 1986). The interaction between light-activated rhodopsin and transducin results in a cascade of effects that give rise to the photoreceptor potential. Evolutionary conservation of a transducin binding site has been postulated, as vertebrate transducin can be activated by vertebrate or invertebrate rhodopsin (Vandenberg and Montal, 1984). On the cytoplasmic face, the first and the third cytoplasmic loop of the Drosophila Rh3 opsin show a significant similarity to the corresponding regions of the ninaE and Rh2 opsins (see Fig. 5). However, only the first of these loops is conserved between Drosophila and vertebrate opsins (residues 87-94; Fig. 4; KXLRXPXN). The third cytoplasmic loop (Figs. 4 and 5, residues 251-284) contains a 10 amino acid insertion that is common to all Drosophila opsins analyzed to date (O'Tousa et al., 1985; Zuker et al., 1985; Cowman et al., 1986; Montell et al., 1987) but is absent from all vertebrate opsins (Ovchinnikov et al., 1982; Hargrave et al., 1983; Nathans and Hogness, 1983, 1984; Nathans et al., 1986).

We previously suggested that the first cytoplasmic loop of rhodopsin is involved in the interaction of rhodopsin with transducin (Zuker et al., 1985). Figure 8 presents sequence data indicating that this region also shows amino acid conservation between opsins (Drosophila and vertebrate) and the $\beta$-adrenergic receptor $(\beta-\mathrm{AR})$, another signal-transducing molecule that interacts with a G-protein (Lefkowitz et al., 1983). Dixon et al. (1986) have recently shown that bovine rhodopsin and the hamster $\beta$-AR share significant amino acid sequence homology, particularly in the transmembrane domains. Functional conservation of catalytic components used for signal transduction in the amplification of the visual response and in the activation of adenylate cyclase-coupled $\beta$-ARs has recently been demonstrated, in that rhodpsin and $\beta$-AR can be properly phosphorylated by the other's kinase (Benovic et al., 1986). Sequence analyses of the additional Drosophila opsins (see Montell et al., 1987) and the recent isolation of the human color opsin genes (Nathans et al., 1986) have allowed us to place all of these proteins in a single phylogenetic tree. The phylogeny shown in Figure 9 was constructed on the basis of the principle of minimal mutation distances (Wilson, 1985; PAUP program, Illinois Natural History Survey, version 2.4.0). The most parsimonious tree favors the branching order shown. Remarkably, the vertebrate $\beta$-AR is equally related to Drosophila opsins as it is to those of vertebrates. The similarity in the number of mutations needed to account for the divergence of these sequences suggests that a single primordial "opsin-like" molecule was present in an ancestor common to vetebrates and invertebrates, and that it gave rise to vertebrate opsins, invertebrate rhodopsins, and the $\beta$-AR. Future studies involving the isolation and characterization of 
the invertebrate homolog to the $\beta$-AR and other sensory receptors will help us dissect and understand similarities in signaltransduction mechanisms.

\section{References}

Bankier, A. T., and B. G. Barrell (1983) Shotgun DNA sequencing. In Techniques in Nucleic Acid Biochemistry, vol. B5, R. A. Flavell, ed., pp. 1-33, Elsevier, Amsterdam.

Benovic, J. L., F. Mayor, R. L. Somers, M. G. Caron, and R. J. Lefkowitz (1986) Light-dependent phosphorylation of rhodopsin by $\beta$-adrenergic receptor kinase. Nature 321:869-872.

Blumberg, D., and H. F. Lodish (1980) Complexity of nuclear and polysomal RNAs in growing Dictyostelium discoideum cells. Dev. Biol. 78: 268-284

Chung, S., S. M. Landfear, D. D. Blumberg, N. S. Cohen, and H. F. Lodish (1981) Synthesis and stability of developmentally regulated Dictyostelium mRNAs are affected by cell-cell contact and cAMP. Cell 24: 785-797.

Cowman, A. C., C. S. Zuker, and G. M. Rubin (1986) An opsin gene expressed in only one photoreceptor cell type of the Drosophila eye. Cell 44: 705-710.

Dixon, R., B. Kobilka, D. Strader, J. Benovic, H. Dohlman, T. Frielle, M. Bolanowski, C. Bennet, E. Rands, R. Diehl, R. Mumford, E. Slater, I. Sigal, M. G. Caron, R. J. Lefkowitz, and C. D. Strader (1986) Cloning of the gene and cDNA for mammalian $\beta$-adrenergic receptor and homology with rhodopsin. Nature 321: 75-79.

Fain, G. L., and J. E. Lisman (1981) Membrane conductances of photoreceptors. Prog. Biophys. Mol. Biol. 37: 91-147.

Farris, J. C. (1972) Estimating phylogenetic trees from distance matrices. Am. Natur. 106: 645-668.

Fein, A., and E. Szuts (1982) Photoreceptors: Their Role in Vision, Cambridge U. P., Cambridge, UK.

Hafen, E., M. Levine, R. L. Garber, and W. J. Gehring (1983) An improved in situ hybridization method for detection of cellular RNAs in Drosophila tissue sections and its application for localizing transcripts of the homeotic Antennapedia gene complex. EMBO J. 2:617623.

Hardie, R. C. (1983) Functional organization of the fly retina. In Progress in Sensory Physiology 5, D. Ottoson, ed., pp. 1-79, Springer, New York.

Hargrave, P. A. (1982) Rhodopsin chemistry, structure and topography. In Progress in Retinal Research, N. Osborne and G. Chader, eds., pp. 1-51, Oxford U. P., Oxford, UK.

Hargrave, P. A., J. H. McDowell, D. R. Curtis, J. K. Wang, E. Juszczak, S.-L. Fong, J. K. Mohanna Rao, and P. Argos (1983) The structure of bovine rhodopsin. Biophys. Struct. Mech. 9: 235-244.

Harris, W. A., W. S. Stark, and J. A. Walker (1976) Genetic dissection of the photoreceptor system in the compound eye of Drosophila melanogaster. J. Physiol. (Lond.) 256: 415-439.

Hillman, P., S. Hochstein, and B. Minke (1983) Transduction in invertebrate photoreceptors: Role of pigment bistability. Physiol. Rev. 63: $668-772$.

Kühn, H. (1984) Interactions between photoexcited rhodopsin and light-activated enzymes in rods. In Progress in Retinal Research, vol. 3, N. Osborne and J. Chader, eds., pp. 124-156, Oxford U. P., Oxford, UK.

Kyte, J., and R. F. Doolittle (1982) A simple model for displaying the hydropathic character of a protein. J. Mol. Biol. 157: 105-132.

Langer-Sofer, P. R., M. Levine, and D. C. Ward (1982) Immunological method for mapping genes on Drosophila polytene chromosomes. Proc. Natl. Acad. Sci. USA 79: 4381-4385.

Lefkowitz, R. L., J. M. Stadel, and M. G. Caron (1983) Adenylate cyclase-coupled beta-adrenergic receptors: Structure and mechanisms of activation and desensitization. Annu. Rev. Biochem. 52: 159-186.

Levy, L. S., R. Ganguly, N. Ganguly, and J. E. Manning (1982) The selection, expression, and organization of a set of head-specific genes in Drosophila. Dev. Biol. 94: 451-464.
Maniatis, T., R. C. Hardison, E. Lacy, J. Lauer, C. O'Connell, D. Quon, G. K. Sim, and A. Efstratiadis (1978) The isolation of structural genes from libraries of eukaryotic DNA. Cell 15: 687-701.

Maniatis, T., E. F. Fritsch, and J. Sambrook (1982) Molecular Cloning: A Laboratory Manual, Cold Spring Harbor Laboratory, Cold Spring Harbor, NY.

Montell, C., K. Jones, C. Zuker, and G. Rubin (1987) A second opsin gene expressed in the ultraviolet-sensitive $\mathrm{R} 7$ photoreceptor cells of Drosophila melanogaster. J. Neurosci. 7: 1558-1566.

Nathans, J., and D. S. Hogness (1983) Isolation, sequence analysis, and intron-exon arrangement of the gene encoding bovine rhodopsin. Cell 34: 807-814.

Nathans, J., and D. S. Hogness (1984) Isolation and nucleotide sequence of the gene encoding human rhodopsin. Proc. Natl. Acad. Sci. USA 81: 4851-4855.

Nathans, J., D. Thomas, and D. S. Hogness (1986) Molecular genetics of human color vision: The genes encoding blue, green and red pigments. Science 232: 193-202.

O'Hare, K., R. Levis, and G. M. Rubin (1983) Transcription of the white locus in Drosophila melanogaster. Proc. Natl. Acad. Sci. USA 80: 6917-6921.

Oliver, D. V., and J. P. Phillips (1970) Technical note. Drosophila Information Service 45: 58.

Ostroy, S. E., M. Wilson, and W. L. Pak (1974) Drosophila rhodopsin: Photochemistry, extraction and differences in the norp $A^{p 12}$ phototransduction mutant. Biochem. Biophys. Res. Commun. 59: 960966.

O'Tousa, J. E., W. Baehr, R. L. Martin, J. Hirsh, W. L. Pak, and M. L. Applebury (1985) The Drosophila ninaE gene encodes an opsin. Cell 40: 839-850.

Ovchinnikov, Y. A. (1982) Rhodopsin and bacteriorhodopsin: Structure-function relationships. FEBS Lett. 148: 179-189.

Ovchinnikov, Y. A., N. G. Abdulaev, M. V. Feigina, I. D. Artamonov, A. S. Zolotarev, A. I. Miroshnikov, U. I. Martynov, M. B. Kustina, A. B. Kudelin, and A. S. Bogachuk (1982) The complete amino acid sequence of visual rhodopsin. Bioorg. Khim. 8: 1011-1014.

Pak, W. L. (1979) Study of photoreceptor function using Drosophila mutants. In Neurogenetics, Genetic Approaches to the Nervous System, X. D. Breakfield, ed., pp 67-99, Elsevier North-Holland, New York.

Pappin, D. J. C., and .J. B. C. Findlay (1984) Sequence variability in the retinal attachment domain of mammalian rhodopsins. Biochem. J. 217: 605-613.

Rubin, G. M. (1985) P transposable elements and their use as genetic tools in Drosophila. Trends Neurosci. 8: 231-233.

Sanger, F., S. Nicklen, and A. R. Coulson (1977) DNA sequencing with chain terminating inhibitors. Proc. Natl. Acad. Sci. USA 74: $5463-5467$

Scavarda, N. J., J. O'Tousa, and W. L. Pak (1983) Drosophila locus with gene-dosage effects on rhodopsin. Proc. Natl. Acad. Sci. USA 80: 4441-4445.

Stieve, H. (1986) The Molecular Mechanism of Photoreception, (Dahlem Konferenzen 1986), H. Stieve, ed., pp 1-10, Springer, Berlin.

Stryer, L. (1983) Transducin and the cyclic GMP phosphodiesterase: Amplifyer proteins in vision. Cold Spring Harbor Symp. Quant. Biol. 48: 841-852.

Stryer, L. (1985) Molecular design of an amplification cascade in vision. Biopolymers $24: 29-47$.

Tomlinson, A. (1985) The cellular dynamics of pattern formation in the eye of Drosophila. J. Embryol. Exp. Morphol. 89: 313-331.

Vandenberg, C., and M. Montal (1984) Light-regulated biochemical events in invertebrate photoreceptors. Biochemistry 23: 2339-2347.

Wilson, A. C. (1985) The molecular basis of evolution. Sci. Am. 253: 164-173.

Zuker, C. S., A. F. Cowman, and G. M. Rubin (1985) Isolation and structure of a rhodopsin gene from $D$. melanogaster. Cell 40: 851858 . 